\title{
Dynamical behavior of stochastic SIRS model with two different incidence rates and Markovian switching
}

\author{
Feng Wang ${ }^{1,2}$ (D) and Zaiming Liu' ${ }^{1 *}$
}

${ }^{\text {*Correspondence: }}$
math_Izm@csu.edu.cn
${ }^{1}$ School of Mathematics and
Statistics, Central South University,
Changsha, P.R. China
Full list of author information is
available at the end of the article

available at the end of the article

\begin{abstract}
In this paper, we discuss SIRS models with two different incidence rates and Markovian switching. First, we consider that the parameters are perturbed by random environment modulated by Markovian switching. The segment method is used to prove that the model has a unique solution and the estimate of the solution is provided. The threshold values for determining extinction or persistence in mean of diseases are presented by theoretical analysis and some inequalities techniques. Furthermore, some results reveal that stochastic disturbances can suppress the disease outbreak. Because of regime switching, the diseases will be extinct (or persistent) although they might be persistent (or extinct) in some certain environments. Then, the model in which incidence rate functions are perturbed by random environment is also discussed and the values to judge the disease extinction are obtained. At last, a few examples are set to illustrate these interesting phenomena, and their simulations have been carried out to verify our theoretical outcomes.
\end{abstract}

Keywords: Stochastic SIRS model; Two incidence rates; Extinction and persistence; Markovian switching

\section{Introduction}

The epidemic model plays a significant role in the spread and control of diseases. Owing to its great importance, it attracts great attention of scholars and has been extensively investigated. The SIRS model, in which the infective individual loses immunity and returns to the susceptive one, is one of its extensions. Also, the epidemic model has other extensions based on different mechanisms and incidence functions. Because life is full of randomness and the parameters involved in the models are affected to a greater or lesser extent by random factors, stochastic models with white noise depicted by Brownian motion have been extensively studied, see [1-7]. In [1], Cai et al. have proved an interesting fact that stochastic fluctuations can suppress disease outbreak, which indicates that stochastic perturbation has an important influence on the behavior of a system.

Thanks to the richness of the research content, epidemic models have been extensively studied in terms of extinction, persistence, stability, stationary distribution, and so on, and a large amount of literature has been published, see [8-14] and the references therein. For example, Bao and Zhang in [10] studied the extinction, persistence, and stationary dis-

(c) The Author(s) 2019. This article is distributed under the terms of the Creative Commons Attribution 4.0 International License (http://creativecommons.org/licenses/by/4.0/), which permits unrestricted use, distribution, and reproduction in any medium, provided you give appropriate credit to the original author(s) and the source, provide a link to the Creative Commons license, and indicate if changes were made. 
tribution of stochastic SIRS models with information intervention, and the study showed that information intervention had a significant influence on the behavior of a disease.

In order to grasp some essential characteristics of life, researchers have studied many different models to fit the reality better. One of them is to turn one disease into two kinds of diseases. Such consideration is reasonable, for instance, children are susceptible to influenza and chickenpox in spring. For this type, epidemic models with two incidence functions have been studied by many researchers, see [13, 15-17] and the references therein.

Owing to the above reasons, Chang et al. in [15] investigated a stochastic SIRS model with two different incidence rates with the following forms:

$$
\left\{\begin{aligned}
\dot{S}(t)= & \left(A-\mu S(t)-\frac{\beta_{1} S(t) I_{1}(t)}{\alpha_{1}+I_{1}(t)}-\frac{\beta_{2} S(t) I_{2}(t)}{\alpha_{2}+S(t)}+\delta R(t)\right) d t \\
& -\frac{\sigma_{1} S(t) I_{1}(t)}{\alpha_{1}+I_{1}(t)} d B_{1}(t)-\frac{\sigma_{2} S(t) I_{2}(t)}{\alpha_{2}+S(t)} d B_{2}(t) \\
\dot{I}_{1}(t)= & \left(\frac{\beta_{1} S(t) I_{1}(t)}{\alpha_{1}(t)}-\left[\mu+d_{1}+r_{1}\right] I_{1}(t)\right) d t+\frac{\sigma_{1} S(t) I_{1}(t)}{\alpha_{1}+I_{1}(t)} d B_{1}(t), \\
\dot{I}_{2}(t)= & \left(\frac{\beta_{2} S(t) I_{2}(t)}{\alpha_{2}+S(t)}-\left[\mu+d_{2}+r_{2}\right] I_{2}(t)\right) d t+\frac{\sigma_{2} S(t) I_{2}(t)}{\alpha_{2}+S(t)} d B_{2}(t), \\
\dot{R}(t)= & \left(r_{1} I_{1}(t)+r_{2} I_{2}(t)-[\mu+\delta] R(t)\right) d t
\end{aligned}\right.
$$

where $S(t), I_{1}(t), I_{2}(t), R(t)$ denote the population sizes of the susceptible, the infected caused by disease 1 , the infected caused by disease 2 , and the removed individuals at time $t$, respectively. $N(t)=S(t)+I_{1}(t)+I_{2}(t)+R(t)$ stands for the total population. The meanings of the parameters in model (1) are expressed as follows: $A$ is the recruitment rate of population, $\mu$ means the natural mortality rate, $\beta_{1}$ and $\beta_{2}$ are contact rates, $\delta$ denotes the rate at which the removed individuals lose their immunity and return to the susceptible population, $d_{1}$ and $d_{2}$ stand for the death rates caused by disease 1 and disease 2, respectively. $r_{1}$ and $r_{2}$ represent the recovery rates of diseases. $\frac{\beta_{1} S(t) I_{1}(t)}{\alpha_{1}+I_{1}(t)}$ and $\frac{\beta_{2} S(t) I_{2}(t)}{\alpha_{2}+S(t)}$ are two incidence rates for two diseases. $B_{i}(t)$ is the standard Brownian motion with the intensity $\sigma_{i}, i=1,2$. The authors obtained two threshold values $R_{1}^{*}$ and $R_{2}^{*}$ which determine the extinction and permanence of two diseases, where $R_{1}^{*}=\frac{\beta_{1} A}{\mu \alpha_{1}\left(\mu+d_{1}+r_{1}\right)}-\frac{\sigma_{1}^{2} A^{2}}{2 \mu^{2} \alpha_{1}^{2}\left(\mu+d_{1}+r_{1}\right)}$ and $R_{2}^{*}=\frac{\beta_{2} A}{\left(\mu \alpha_{2}+A\right)\left(\mu+d_{2}+r_{2}\right)}-\frac{\sigma_{2}^{2} A^{2}}{2\left(\mu \alpha_{2}+A\right)^{2}\left(\mu+d_{2}+r_{2}\right)}$.

However, the above stochastic model is investigated in a fixed environment. The reality is that the environment is also changing. Taking the example above into account, the spread of diseases in children is affected by seasons and temperature, the mechanisms of the effects of spring and autumn on disease are obviously different. Such changes of the environment cannot be described by the white noise driven by Brownian motions. Thus, the model containing both dynamics and discrete events in which the events are modulated by continuous time Markov chain is reasonable. The epidemic models with Markovian switching have been investigated by many scholars, see [18-23]. There is a lot of literature about other systems modulated by Markovian switching, see [24-29]. For more theory and details about Markovian switching, we refer the readers to [30, 31].

To our best knowledge, there is little literature to study a stochastic SIRS model with two incidence rates and Markovian switching. In this paper, we first discuss the model as 
follows:

$$
\left\{\begin{aligned}
\dot{S}(t)= & \left(A\left(\Lambda_{t}\right)-\mu\left(\Lambda_{t}\right) S(t)-\frac{\beta_{1}\left(\Lambda_{t}\right) S(t) I_{1}(t)}{\alpha_{1}+I_{1}(t)}-\frac{\beta_{2}\left(\Lambda_{t}\right) S(t) I_{2}(t)}{\alpha_{2}+S(t)}+\delta\left(\Lambda_{t}\right) R(t)\right) d t \\
& -\frac{\sigma_{1}\left(\Lambda_{t}\right) S(t) I_{1}(t)}{\alpha_{1}+I_{1}(t)} d B_{1}(t)-\frac{\sigma_{2}\left(\Lambda_{t}\right) S(t) I_{2}(t)}{\alpha_{2}+S(t)} d B_{2}(t), \\
\dot{I}_{1}(t)= & \left(\frac{\beta_{1}\left(\Lambda_{t}\right) S(t) I_{1}(t)}{\alpha_{1}+I_{1}(t)}-\left[\mu\left(\Lambda_{t}\right)+d_{1}\left(\Lambda_{t}\right)+r_{1}\left(\Lambda_{t}\right)\right] I_{1}(t)\right) d t+\frac{\sigma_{1}\left(\Lambda_{t}\right) S(t) I_{1}(t)}{\alpha_{1}+I_{1}(t)} d B_{1}(t), \\
\dot{I}_{2}(t)= & \left(\frac{\beta_{2}\left(\Lambda_{t}\right) S(t) I_{2}(t)}{\alpha_{2}+S(t)}-\left[\mu\left(\Lambda_{t}\right)+d_{2}\left(\Lambda_{t}\right)+r_{2}\left(\Lambda_{t}\right)\right] I_{2}(t)\right) d t+\frac{\sigma_{2}\left(\Lambda_{t}\right) S(t) I_{2}(t)}{\alpha_{2}+S(t)} d B_{2}(t), \\
\dot{R}(t)= & \left(r_{1}\left(\Lambda_{t}\right) I_{1}(t)+r_{2}\left(\Lambda_{t}\right) I_{2}(t)-\left[\mu\left(\Lambda_{t}\right)+\delta\left(\Lambda_{t}\right)\right] R(t)\right) d t,
\end{aligned}\right.
$$

where $\left(\Lambda_{t}\right)$ is a continuous-time Markov chain, taking values in a finite state space $\mathcal{N}=$ $\{1,2, \ldots, N\}$ and with generator $Q=\left(q_{i j}\right)_{N \times N}$, which means that

$$
\mathbb{P}\left(\Lambda_{t+\theta}=j \mid \Lambda_{t}=i\right)= \begin{cases}q_{i j} \theta+o(\theta) & \text { if } i \neq j, \\ 1+q_{i i} \theta+o(\theta) & \text { if } i=j,\end{cases}
$$

supposing that $\theta \downarrow 0$. Throughout this paper, we assume that the $Q$ matrix is conservative and irreducible. Then, according to the theory of Markov chain, we obtain that $\left(\Lambda_{t}\right)$ has a unique stationary distribution $\left(\pi_{i}\right)$ satisfying the equations

$$
\pi Q=0, \quad \sum_{i=1}^{N} \pi_{i}=1 .
$$

On the other hand, the incidence functions may also be affected in different environments besides the influence of parameters in the above model. Hence, a new SIRS model in which the incidence functions vary depending on different environments is proposed as follows:

$$
\left\{\begin{aligned}
\dot{S}(t)= & \left(A\left(\Lambda_{t}\right)-\mu\left(\Lambda_{t}\right) S(t)-\frac{\beta_{1}\left(\Lambda_{t}\right) S(t) I_{1}(t)^{\Lambda_{t}}}{\alpha_{1}+I_{1}(t)}-\frac{\beta_{2}\left(\Lambda_{t}\right) S(t) I_{2}(t)^{\Lambda_{t}}}{\alpha_{2}+S(t)}+\delta\left(\Lambda_{t}\right) R(t)\right) d t \\
& -\frac{\sigma_{1}\left(\Lambda_{t}\right) S(t) I_{1}(t)^{\Lambda_{t}}}{\alpha_{1}+I_{1}(t)} d B_{1}(t)-\frac{\sigma_{2}\left(\Lambda_{t}\right) S(t) I_{2}(t) \Lambda^{\Lambda_{t}}}{\alpha_{2}+S(t)} d B_{2}(t), \\
\dot{I}_{1}(t)= & \left(\frac{\beta_{1}\left(\Lambda_{t}\right) S(t) I_{1}(t) \Lambda_{t}}{\alpha_{1}+I_{1}(t)}-\left[\mu\left(\Lambda_{t}\right)+d_{1}\left(\Lambda_{t}\right)+r_{1}\left(\Lambda_{t}\right)\right] I_{1}(t)\right) d t \\
& +\frac{\sigma_{1}\left(\Lambda_{t}\right) S(t) I_{1}(t) \Lambda_{t}}{\alpha_{1}+I_{1}(t)} d B_{1}(t) \\
\dot{I}_{2}(t)= & \left(\frac{\beta_{2}\left(\Lambda_{t}\right) S(t) I_{2}(t) \Lambda_{t}}{\alpha_{2}+S(t)}-\left[\mu\left(\Lambda_{t}\right)+d_{2}\left(\Lambda_{t}\right)+r_{2}\left(\Lambda_{t}\right)\right] I_{2}(t)\right) d t \\
& +\frac{\sigma_{2}\left(\Lambda_{t}\right)(t) I_{2}(t) \Lambda^{\Lambda_{t}}}{\alpha_{2}+S(t)} d B_{2}(t) \\
\dot{R}(t)= & \left(r_{1}\left(\Lambda_{t}\right) I_{1}(t)+r_{2}\left(\Lambda_{t}\right) I_{2}(t)-\left[\mu\left(\Lambda_{t}\right)+\delta\left(\Lambda_{t}\right)\right] R(t)\right) d t .
\end{aligned}\right.
$$

Although there are some epidemic models with two incidence functions or Markovian switching, and some methods are used to solve different problems, for example, Markov semigroup theory was used in [23] to obtain the existence of stationary distribution, the stochastic SIRS model (2), which combines two non-linear incidence functions and Markov switching, has rarely been proposed and studied. In order to overcome the difficulties caused by two factors, some new inequality techniques are utilized to study the asymptotic behavior of diseases. In addition, model (4), in which the incidence functions are affected by Markov chain, has rarely been studied. The methods and results of these models can enrich epidemiological theories and help control the spread of diseases.

The rest of the paper is arranged as follows. Section 2 provides some background knowledge and proves that model (2) has a unique positive solution. Section 3 focuses on the 
conditions under which the two diseases will be extinct and presents the threshold values for judging disease extinction. Section 4 proceeds with the threshold values to determine the disease persistence. Section 5 studies the influence of Markovian switching upon incidence functions in model (4) and gives the conditions to judge disease extinction. Section 6 presents some examples and their figures to illustrate our theoretical results; and finally, Sect. 7 gives the conclusions and future research of current work.

\section{Background knowledge}

In this section, we present a number of definitions, notations, and lemmas which will be used later in the article.

Let $\check{A}=\max _{i \in \mathcal{N}} A(i), \hat{A}=\min _{i \in \mathcal{N}} A(i), \check{\beta_{1}}=\max _{i \in \mathcal{N}} \beta_{1}(i)$, and $\hat{\beta}_{1}=\min _{i \in \mathcal{N}} \beta_{1}(i)$. Similarly, for the parameters $\check{\mu}, \hat{\mu}, \check{\beta}_{2}, \hat{\beta_{2}}, \hat{\delta}, \check{r_{1}}, \check{r_{2}}, \hat{\sigma}_{1}$, we define them in the same way.

Throughout this paper, let $\left(\Omega, \mathcal{F},\{\mathcal{F}\}_{t \geq 0}, \mathbb{P}\right)$ be a complete probability space with a filtration $\{\mathcal{F}\}_{t \geq 0}$ satisfying the usual conditions. Take into account the SDEs of the following form:

$$
d X(t)=b\left(X, \Lambda_{t}\right) d t+\sigma\left(X, \Lambda_{t}\right) d B(t)
$$

where $b: \mathbb{R}^{n} \times \mathcal{N} \rightarrow \mathbb{R}^{n}, \sigma: \mathbb{R}^{n} \times \mathcal{N} \rightarrow \mathbb{R}^{n \times m}$, and $B(t)=\left(B_{t}^{1}, \ldots, B_{t}^{m}\right)^{T}$ is $m$-dimensional standard Brownian motion defined on the complete probability space. For $i \in \mathcal{N}$ and any twice continuous-differentiable function $V(X, i)$, define the operator $\mathcal{L}$ by

$$
\mathcal{L} V(X, i)=V_{x}(X, i) b(X, i)+\frac{1}{2} \operatorname{tr}\left[\sigma^{T}(X, t) V_{x x} \sigma(X, t)\right]+\sum_{j=1}^{N} q_{i j} V(X, j) .
$$

To proceed, we give the definitions for describing the behavior of the diseases. Let $\mathbb{R}_{+}^{4}=$ $\left\{x_{i}>0, i=1,2,3,4\right\}$, and for an integrable function $f$, define the notation $\langle f(t)\rangle$ by $\langle f(t)\rangle=$ $\frac{1}{t} \int_{0}^{t} f(r) d r$.

\section{Definition 2.1}

- The disease $I(t)$ is called extinct if $\lim _{t \rightarrow \infty} I(t)=0$;

- The disease $I(t)$ is called persistent if there exists a positive constant $\lambda$ such that $\liminf _{t \rightarrow \infty}\langle f(t)\rangle \geq \lambda$.

Lemma 2.1 For any initial value $\left(S(0), I_{1}(0), I_{2}(0), R(0)\right) \in \mathbb{R}_{+}^{4}$, and $i_{0} \in \mathcal{N}$, model (2) has a unique solution $\left(S(t), I_{1}(t), I_{2}(t), R(t)\right)$ for $t>0$, and the solution remains in $R_{+}^{4}$ with probability 1. Moreover, the solution satisfies the inequality

$$
N(t) \leq N(0) e^{-\int_{0}^{t} \mu\left(\Lambda_{s}\right) d s}+\int_{0}^{t} A\left(\Lambda_{s}\right) e^{-\int_{s}^{t} \mu\left(\Lambda_{r}\right) d r} d s, \quad \text { a.s. }
$$

then, obviously,

$$
N(t) \leq N(0) e^{-\hat{\mu} t}+\frac{\check{A}}{\hat{\mu}} \leq N(0)+\frac{\check{A}}{\hat{\mu}}, \quad \text { a.s. },
$$

holds. 
Proof The existence and uniqueness of the solution to model (2) can be obtained by the segment method. Precisely, let $0=t_{0}<t_{1}<\cdots<t_{n}<\cdots$ be the set of all jumping times of the Markov chain $\left(\Lambda_{t}\right)$. Then, for $t \in\left[0, t_{1}\right), \Lambda_{t}=i_{0}$. The model is simplified as follows:

$$
\left\{\begin{aligned}
\dot{S}(t)= & \left(A\left(i_{0}\right)-\mu\left(i_{0}\right) S(t)-\frac{\beta_{1}\left(i_{0}\right) S(t) I_{1}(t)}{\alpha_{1}+I_{1}(t)}-\frac{\beta_{2}\left(i_{0}\right) S(t) I_{2}(t)}{\alpha_{2}+S_{1}(t)}+\delta\left(i_{0}\right) R(t)\right) d t \\
& -\frac{\sigma_{1}\left(i_{0}\right) S(t) I_{1}(t)}{\alpha_{1}+I_{1}(t)} d B_{1}(t)-\frac{\sigma_{2}\left(i_{0}\right) S(t) I_{2}(t)}{\alpha_{2}+S(t)} d B_{2}(t), \\
\dot{I}_{1}(t)= & \left(\frac{\beta_{1}\left(i_{0}\right) S(t) I_{1}(t)}{\alpha_{1}+I_{1}(t)}-\left[\mu\left(i_{0}\right)+d_{1}\left(i_{0}\right)+r_{1}\left(i_{0}\right)\right] I_{1}(t)\right) d t+\frac{\sigma_{1}\left(i_{0}\right) S(t) I_{1}(t)}{\alpha_{1}+I_{1}(t)} d B_{1}(t), \\
\dot{I}_{2}(t)= & \left(\frac{\beta_{2}\left(i_{0}\right) S(t) I_{2}(t)}{\alpha_{2}+S(t)}-\left[\mu\left(i_{0}\right)+d_{2}\left(i_{0}\right)+r_{2}\left(i_{0}\right)\right] I_{2}(t)\right) d t+\frac{\sigma_{2}\left(i_{0}\right) S(t) I_{2}(t)}{\alpha_{2}+S(t)} d B_{2}(t), \\
\dot{R}(t)= & \left(r_{1}\left(i_{0}\right) I_{1}(t)+r_{2}\left(i_{0}\right) I_{2}(t)-\left[\mu\left(i_{0}\right)+\delta\left(i_{0}\right)\right] R(t)\right) d t .
\end{aligned}\right.
$$

With regard to model (8), the existence and uniqueness of a positive solution can be obtained by constructing the Lyapunov function $V\left(S, I_{1}, I_{2}, R\right)=-\ln \frac{S}{h_{1}}-\ln \frac{I_{1}}{h_{1}}-\ln \frac{I_{2}}{h_{1}}-\ln \frac{R}{h_{1}}$, where the constant $h_{1}=\max \left\{N(0), \frac{A}{\mu}\right\}$. For more details, please refer to the literature [15]. Then, for $t \in\left[t_{1}, t_{2}\right), \Lambda_{t}=\Lambda_{t_{1}} \in \mathcal{N}$, we use the same Lyapunov function above to acquire the positive solution. Repeating this procedure, we can arrive at the conclusion that the model has the unique positive solution with the initial value $\left(S(0), I_{1}(0), I_{2}(0), R(0)\right)$.

Then we prove inequality (6). Adding up all formulas of (2) yields that

$$
\begin{aligned}
d N(t) & =\left[A\left(\Lambda_{t}\right)-\mu\left(\Lambda_{t}\right) N(t)-d_{1}\left(\Lambda_{t}\right) I_{1}(t)-d_{2}\left(\Lambda_{t}\right) I_{2}(t)\right] d t \\
& \leq\left[A\left(\Lambda_{t}\right)-\mu\left(\Lambda_{t}\right) N(t)\right] d t
\end{aligned}
$$

The last equality holds thanks to $I_{i}(t) \geq 0, i=1,2$. Hence, we can obtain that

$$
\frac{d N(t)}{d t} \leq A\left(\Lambda_{t_{k}}\right)-\mu\left(\Lambda_{t_{k}}\right) N(t), \quad t \in\left[t_{k}, t_{k+1}\right)
$$

Using the method of variation of constant, we can get the following formula:

$$
\begin{aligned}
N(t) & \leq N\left(t_{k}\right) e^{-\mu\left(\Lambda_{t_{k}}\right)\left(t-t_{k}\right)}+\int_{t_{k}}^{t} A\left(\Lambda_{s}\right) e^{-\mu\left(\Lambda_{t_{k}}\right)(t-s)} d s \\
& =N\left(t_{k}\right) e^{-\int_{t_{k}}^{t} \mu\left(\Lambda_{s}\right) d s}+\int_{t_{k}}^{t} A\left(\Lambda_{s}\right) e^{-\int_{s}^{t} \mu\left(\Lambda_{r}\right) d r} d s .
\end{aligned}
$$

By the recursive rule, letting $t=t_{k}$, we get that

$$
N\left(t_{k}\right) \leq N\left(t_{k-1}\right) e^{-\int_{t_{k-1}}^{t_{k}} \mu\left(\Lambda_{s}\right) d s}+\int_{t_{k-1}}^{t_{k}} A\left(\Lambda_{s}\right) e^{-\int_{s}^{t} \mu\left(\Lambda_{r}\right) d r} d s
$$

Inserting (11) into (10) and repeating this calculation yield that

$$
\begin{aligned}
N(t) & \leq N\left(t_{k-1}\right) e^{-\int_{t_{k-1}}^{t} \mu\left(\Lambda_{s}\right) d s}+\int_{t_{k-1}}^{t} A\left(\Lambda_{s}\right) e^{-\int_{s}^{t} \mu\left(\Lambda_{r}\right) d r} d s \\
& \leq \cdots \\
& \leq N(0) e^{-\int_{0}^{t} \mu\left(\Lambda_{s}\right) d s}+\int_{0}^{t} A\left(\Lambda_{s}\right) e^{-\int_{s}^{t} \mu\left(\Lambda_{r}\right) d r} d s
\end{aligned}
$$


and

$$
\begin{aligned}
N(t) & \leq N(0) e^{-\int_{0}^{t} \hat{\mu} d s}+\int_{0}^{t} \check{A} e^{-\int_{s}^{t} \hat{\mu} d r} d s \\
& =N(0) e^{-\hat{\mu} t}+\frac{\check{A}}{\hat{\mu}}\left(1-e^{-\hat{\mu} t}\right) .
\end{aligned}
$$

Hence, (6) and (7) are verified.

\section{Extinction of diseases}

In this section, we study some sufficient conditions to justify the extinction of the diseases of model (2). To begin with, we present some lemmas which will be used in the paper.

Lemma 3.1 For the solution of model (2) with any initial value, it holds that

$$
\limsup _{t \rightarrow \infty} R(t) \leq \frac{\check{r_{1}}+\check{r_{2}}}{\hat{\mu}+\hat{\delta}} \limsup _{t \rightarrow \infty}\left(I_{1}(t)+I_{2}(t)\right) .
$$

Proof From the fourth equation of model (2), we deduce that

$$
\frac{d R(t)}{d t} \leq\left(\check{r_{1}}+\check{r_{2}}\right)\left(I_{1}(t)+I_{2}(t)\right)-(\hat{\mu}+\hat{\delta}) R(t)
$$

Then integrating the above formula and letting $I(t)=I_{1}(t)+I_{2}(t)$ yield that

$$
\begin{aligned}
R(t) & \leq R(0) e^{-(\hat{\mu}+\hat{\delta}) t}+\left(\check{r_{1}}+\check{r_{2}}\right) \int_{0}^{t} e^{-(\hat{\mu}+\hat{\delta})(t-s)}\left(I_{1}(s)+I_{2}(s)\right) d s \\
& =R(0) e^{-(\hat{\mu}+\hat{\delta}) t}+\left(\check{r_{1}}+\check{r_{2}}\right) \int_{0}^{t} e^{-(\hat{\mu}+\hat{\delta}) u} I(t-u) d u \\
& \leq R(0) e^{-(\hat{\mu}+\hat{\delta}) t}+\left(\check{r_{1}}+\check{r_{2}}\right) \int_{0}^{\infty} e^{-(\hat{\mu}+\hat{\delta}) u} I(t-u) d u .
\end{aligned}
$$

By Fatou's lemma, we can get

$$
\begin{aligned}
\limsup _{t \rightarrow \infty} R(t) & \leq \limsup _{t \rightarrow \infty} R(0) e^{-(\hat{\mu}+\hat{\delta}) t}+\limsup _{t \rightarrow \infty}\left(\check{r_{1}}+\check{r_{2}}\right) \int_{0}^{\infty} e^{-(\hat{\mu}+\hat{\delta}) u} I(t-u) d u \\
& \leq\left(\check{r_{1}}+\check{r_{2}}\right) \int_{0}^{\infty} \limsup _{t \rightarrow \infty} I(t-u) e^{-(\hat{\mu}+\hat{\delta}) u} d u \\
& =\left(\check{r_{1}}+\check{r_{2}}\right) \limsup _{t \rightarrow \infty} I(t) \int_{0}^{\infty} e^{-(\hat{\mu}+\hat{\delta}) u} d u \\
& =\frac{\check{r_{1}}+\check{r_{2}}}{\hat{\mu}+\hat{\delta}} \limsup _{t \rightarrow \infty}\left(I_{1}(t)+I_{2}(t)\right) .
\end{aligned}
$$

Lemma 3.2 For the solution of model (2) with any initial value, the following formulas hold:

$$
\begin{array}{ll}
\lim _{t \rightarrow \infty} \frac{1}{t} \int_{0}^{t} \frac{\sigma_{1}\left(\Lambda_{s}\right) S(s)}{\alpha_{1}+I_{1}(s)} d B_{1}(s)=0, & \lim _{t \rightarrow \infty} \frac{1}{t} \int_{0}^{t} \frac{\sigma_{2}\left(\Lambda_{s}\right) S(s)}{\alpha_{2}+S(s)} d B_{2}(s)=0, \\
\lim _{t \rightarrow \infty} \frac{1}{t} \int_{0}^{t} \sigma_{1}\left(\Lambda_{s}\right) S(s) d B_{1}(s)=0, & \lim _{t \rightarrow \infty} \frac{1}{t} \int_{0}^{t} \frac{\sigma_{1}\left(\Lambda_{s}\right) S(s) I_{1}(s)^{\Lambda_{s}-1}}{\alpha_{1}+I_{1}(s)} d B_{1}(s)=0,
\end{array}
$$




$$
\lim _{t \rightarrow \infty} \frac{1}{t} \int_{0}^{t} \frac{\sigma_{2}\left(\Lambda_{s}\right) S(s) I_{2}(s)^{\Lambda_{s}-1}}{\alpha_{2}+S(s)} d B_{2}(s)=0, \quad \text { a.s. }
$$

Proof We only give the proof of the first formula and the fourth one above, the other three formulas can be proved in a similar way.

Let $M(t)=\int_{0}^{t} \frac{\sigma_{1}\left(\Lambda_{s}\right) S(s)}{\alpha_{1}+I_{1}(s)} d B_{1}(s), A(t)=\int_{0}^{t} \frac{\sigma_{1}^{2}\left(\Lambda_{s}\right) S^{2}(s)}{\left(\alpha_{1}+I_{1}(s)\right)^{2}} d s$, and $p>2$. Making use of the BDG inequality, one can get

$$
\mathbb{E}\left[\sup _{0 \leq s \leq t}|M(s)|^{p}\right] \leq C_{p} \mathbb{E}[A(t)]^{\frac{p}{2}} \leq C_{p} \mathbb{E}\left[\sup _{0 \leq s \leq t} \frac{\sigma_{1}^{2}\left(\Lambda_{s}\right) S^{2}(s)}{\left(\alpha_{1}+I_{1}(s)\right)^{2}}\right]^{\frac{p}{2}} t^{\frac{p}{2}} \leq \tilde{C}_{p} t^{\frac{p}{2}},
$$

where $\tilde{C}_{p}=C_{p}\left(\frac{\check{\sigma_{1}(N(0) \hat{\mu}+\check{A})}}{\alpha_{1} \hat{\mu}}\right)^{p}$.

Let $k \geq 1$ be a positive integer and $\epsilon$ be an arbitrarily small positive constant. By Chebyshev's inequality, one has

$$
\begin{aligned}
\mathbb{P}\left\{\sup _{k \theta \leq t \leq(k+1) \theta}|M(t)|^{p}>(k \theta)^{1+\epsilon+\frac{p}{2}}\right\} & \leq \frac{\mathbb{E}|M((k+1) \theta)|^{p}}{(k \theta)^{1+\epsilon+\frac{p}{2}}} \\
& \leq \frac{\tilde{C}_{p}[(k+1) \theta]^{\frac{p}{2}}}{(k \theta)^{1+\epsilon+\frac{p}{2}}} \leq \frac{\tilde{C}_{p}(2 k \theta)^{\frac{p}{2}}}{(k \theta)^{1+\epsilon+\frac{p}{2}}}=\frac{\tilde{C}_{p} 2^{\frac{p}{2}}}{(k \theta)^{1+\epsilon}} .
\end{aligned}
$$

Since $\sum_{k=0}^{\infty} \frac{\tilde{C}_{p} 2^{\frac{p}{2}}}{(k \theta)^{1+\epsilon}}$ converges, the Borel-Cantelli lemma yields that, for almost all $\omega \in \Omega$, there exists a positive integer $n_{0}=n_{0}(\omega)$ such that

$$
\sup _{k \theta \leq t \leq(k+1) \theta}|M(t)|^{p} \leq(k \theta)^{1+\epsilon+\frac{p}{2}}, \quad \text { when } n \geq n_{0} .
$$

For $k \theta \leq t \leq(k+1) \theta$ and $n \geq n_{0}$, we have

$$
\ln |M(t)|^{p} \leq\left(1+\epsilon+\frac{p}{2}\right) \ln t, \quad \text { a.s. }
$$

Because $\epsilon$ is arbitrary, letting $\epsilon \rightarrow 0$, we can obtain

$$
\limsup _{t \rightarrow \infty} \frac{\ln |M(t)|}{\ln t} \leq \frac{1}{2}+\frac{1}{p}, \quad \text { a.s. }
$$

Then, for a sufficiently small constant $\epsilon\left(\epsilon<\frac{1}{2}-\frac{1}{p}\right)$, there exists a constant $N(\omega)$, for $t \geq N(\omega)$, one has

$$
\ln |M(t)| \leq\left(\frac{1}{2}+\frac{1}{p}+\epsilon\right) \ln t, \quad \text { a.s. }
$$

Thus,

$$
\limsup _{t \rightarrow \infty} \frac{|M(t)|}{t} \leq \limsup _{t \rightarrow \infty} \frac{t^{\left(\frac{1}{2}+\frac{1}{p}+\epsilon\right)}}{t}=0, \quad \text { a.s. }
$$

The first formula of (13) has been established. 
Next, we prove that the fourth formula holds. We use the strong law of large numbers to prove it.

Assume that $M_{1}(t)=\int_{0}^{t} \frac{\sigma_{1}\left(\Lambda_{s}\right) S(s) I_{1}(s)^{\Lambda_{s}-1}}{\alpha_{1}+I_{1}(s)} d B_{1}(s)$, then one has

$$
\limsup _{t \rightarrow \infty} \frac{\left\langle M_{1}, M_{1}\right\rangle_{t}}{t} \leq \limsup _{t \rightarrow \infty} \frac{1}{t} \int_{0}^{t}\left(\frac{\check{\sigma_{1}}\left(N(0)+\frac{\check{A}}{\hat{\mu}}\right)^{N}}{\alpha_{1}}\right) d t<\infty, \quad \text { a.s. }
$$

Thus, we get that $\limsup _{t \rightarrow \infty} \frac{M_{1}(t)}{t}=0$.

Lemma 3.3 (Strong ergodicity theorem, cf. [31]) Assume that $\left(X_{t}, \Lambda_{t}\right)$ is positive recurrent and $\pi$ is the stationary distribution of $\left(X_{t}, \Lambda_{t}\right)$, then for any bounded measurable function $f$ defined on $\mathbb{R}_{+} \times \mathcal{N}$, it holds

$$
\lim _{t \rightarrow \infty} \frac{1}{t} \int_{0}^{t} f\left(X_{s}, \Lambda_{s}\right) d s=\sum_{i \in \mathcal{N}} \int_{\mathbb{R}_{+}} f(x, i) \pi(d x, i) .
$$

After introducing several lemmas above, we provide a sufficient condition to judge the extinction of diseases.

\section{Theorem 3.1 If}

$$
\hat{\sigma}_{1}^{2}>\frac{\frac{1}{2} \sum_{i \in \mathcal{N}} \pi_{i} \beta_{1}^{2}(i)}{\sum_{i \in \mathcal{N}} \pi_{i}\left(\mu(i)+d_{1}(i)+r_{1}(i)\right)}
$$

and

$$
\hat{\sigma}_{2}^{2}>\frac{\frac{1}{2} \sum_{i \in \mathcal{N}} \pi_{i} \beta_{2}^{2}(i)}{\sum_{i \in \mathcal{N}} \pi_{i}\left(\mu(i)+d_{2}(i)+r_{2}(i)\right)},
$$

then the two diseases of model (2) will be extinct almost surely, i.e.,

$$
\lim _{t \rightarrow \infty} I_{i}(t)=0, \quad i=1,2 \text {, a.s. }
$$

Proof Applying Itô's formula to $V_{1}(t)=\ln I_{1}(t)$ and the chain rule to the second equation of model (2) yields that

$$
d \ln I_{1}(t)=\mathcal{L} V_{1}(t) d t+\frac{\sigma_{1}\left(\Lambda_{t}\right) S(t)}{\alpha_{1}+I_{1}(t)} d B_{1}(t)
$$

where $\mathcal{L} V_{1}(t)=\frac{\beta_{1}\left(\Lambda_{t}\right) S(t)}{\alpha_{1}+I_{1}(t)}-\left[\mu\left(\Lambda_{t}\right)+d_{1}\left(\Lambda_{t}\right)+r_{1}\left(\Lambda_{t}\right)\right]-\frac{\sigma_{1}^{2}\left(\Lambda_{t}\right) S^{2}(t)}{2\left(\alpha_{1}+I_{1}(t)\right)^{2}}$. Thus,

$$
\begin{aligned}
\mathcal{L} V_{1}(t) & \leq \frac{\beta_{1}\left(\Lambda_{t}\right) S(t)}{\alpha_{1}+I_{1}(t)}-\left[\mu\left(\Lambda_{t}\right)+d_{1}\left(\Lambda_{t}\right)+r_{1}\left(\Lambda_{t}\right)\right]-\frac{\hat{\sigma}_{1}^{2} S^{2}(t)}{2\left(\alpha_{1}+I_{1}(t)\right)^{2}} \\
& =-\frac{\hat{\sigma}_{1}^{2}}{2}\left(\frac{S(t)}{\alpha_{1}+I_{1}(t)}-\frac{\beta_{1}\left(\Lambda_{t}\right)}{\hat{\sigma}_{1}^{2}}\right)^{2}+\frac{\beta_{1}^{2}\left(\Lambda_{t}\right)}{2 \hat{\sigma}_{1}^{2}}-\left[\mu\left(\Lambda_{t}\right)+d_{1}\left(\Lambda_{t}\right)+r_{1}\left(\Lambda_{t}\right)\right] \\
& \leq \frac{\beta_{1}^{2}\left(\Lambda_{t}\right)}{2 \hat{\sigma}_{1}^{2}}-\left[\mu\left(\Lambda_{t}\right)+d_{1}\left(\Lambda_{t}\right)+r_{1}\left(\Lambda_{t}\right)\right] .
\end{aligned}
$$


Integrating from 0 to $t$ for (16) and dividing both sides by $t$, one has

$$
\begin{aligned}
\frac{\ln I_{1}(t)}{t} \leq & \frac{1}{t} \int_{0}^{t}\left(\frac{\beta_{1}^{2}\left(\Lambda_{s}\right)}{2 \hat{\sigma}_{1}^{2}}-\left[\mu\left(\Lambda_{s}\right)+d_{1}\left(\Lambda_{s}\right)+r_{1}\left(\Lambda_{s}\right)\right]\right) d s \\
& +\frac{1}{t} \int_{0}^{t} \frac{\sigma_{1}\left(\Lambda_{s}\right) S(s)}{\alpha_{1}+I_{1}(s)} d B_{1}(s)+\frac{\ln I_{1}(0)}{t} .
\end{aligned}
$$

Letting $t \rightarrow \infty$, using Lemmas 3.2 and 3.3, we get that

$$
\limsup _{t \rightarrow \infty} \frac{\ln I_{1}(t)}{t} \leq \sum_{i \in \mathcal{M}} \pi_{i}\left(\frac{\beta_{1}^{2}(i)}{2 \hat{\sigma}_{1}^{2}}-\left[\mu(i)+d_{1}(i)+r_{1}(i)\right]\right) .
$$

Under the condition of (14), we can obtain that $\lim _{t \rightarrow \infty} I_{1}(t)=0$, a.s. The proof of (15) is the same as that of the first one, we omit it here to avoid repetition.

From conditions (14) and (15), we can see that when the intensities of white noise in each environment are sufficiently large, the two diseases will go to extinction almost surely as time goes on. This reveals the effect of white noise on diseases. Next, we will consider the situation of small intensities of white noise and provide the sufficient condition for extinction of diseases.

Theorem 3.2 For the solution $S(t), I_{1}(t), I_{2}(t), R(t)$ to model (2) with the initial value $S(0), I_{1}(0), I_{2}(0), R(0) \in R_{+}^{4}$, if $\frac{\check{A}}{\alpha_{1} \hat{\mu}} \leq \min _{i \in \mathcal{N}}\left\{\frac{\beta_{1}(i)}{\sigma_{1}^{2}(i)}\right\}, \frac{\check{A}}{\alpha_{2} \hat{\mu}+\check{A}} \leq \min _{i \in \mathcal{N}}\left\{\frac{\beta_{2}(i)}{\sigma_{2}^{2}(i)}\right\}$, and

$$
\begin{aligned}
& \Delta_{1}:=\frac{\sum_{i \in \mathcal{N}} \pi(i) \beta_{1}(i) \frac{\check{A}}{\alpha_{1} \hat{\mu}}}{\sum_{i \in \mathcal{N}} \pi(i)\left[\mu(i)+d_{1}(i)+r_{1}(i)+\frac{\sigma_{1}^{2}(i) \check{A}^{2}}{2 \alpha_{1}^{2} \hat{\mu}^{2}}\right]}<1, \\
& \Delta_{2}:=\frac{\sum_{i \in \mathcal{N}} \pi(i) \beta_{2}(i) \frac{\check{A}}{\alpha_{2} \hat{\mu}+\check{A}}}{\sum_{i \in \mathcal{N}} \pi(i)\left[\mu(i)+d_{2}(i)+r_{2}(i)+\frac{\sigma_{2}^{2}(i) \check{A}^{2}}{2\left(\alpha_{2} \hat{\mu}+\check{A}\right)^{2}}\right]}<1,
\end{aligned}
$$

then

$$
\lim _{t \rightarrow \infty} I_{i}(t)=0, \quad i=1,2, \text { a.s. }
$$

which means that the two diseases will go to extinction.

Also,

$$
\begin{aligned}
& \lim _{t \rightarrow \infty} R(t)=0, \quad \text { a.s. } \\
& \lim _{t \rightarrow \infty} \frac{1}{t} \int_{0}^{t} \mu\left(\Lambda_{s}\right) S(s) d s=\sum_{i \in \mathcal{N}} \pi(i) A(i), \quad \text { a.s. }
\end{aligned}
$$

Proof Applying Itô's formula to $V_{1}=\ln I_{1}(t)$, one has

$$
d \ln I_{1}(t)=\mathcal{L} V_{1} d t+\frac{\sigma_{1}\left(\Lambda_{t}\right) S(t)}{\alpha_{1}+I_{1}(t)} d B_{1}(t)
$$

where $\mathcal{L} V_{1}=\frac{\beta_{1}\left(\Lambda_{t}\right) S(t)}{\alpha_{1}+I_{1}(t)}-\left[\mu\left(\Lambda_{t}\right)+d_{1}\left(\Lambda_{t}\right)+r_{1}\left(\Lambda_{t}\right)\right]-\frac{\sigma_{1}^{2}\left(\Lambda_{t}\right) S^{2}(t)}{2\left(\alpha_{1}+I_{1}(t)\right)^{2}}$. 
Let $x(t)=\frac{S(t)}{\alpha_{1}+I_{1}(t)}$, then $x(t) \leq \frac{1}{\alpha_{1}}\left(N(0) e^{-\hat{\mu} t}+\frac{\check{A}}{\hat{\mu}}\right)$, and $\mathcal{L} V_{1}$ can be regarded as a combination of $N$ quadratic functions. Each function is increasing in the interval $\left[0, \frac{\beta_{1}\left(\Lambda_{t}\right)}{\sigma_{1}^{2}\left(\Lambda_{t}\right)}\right]$. Therefore, when $\frac{\check{A}}{\alpha_{1} \hat{\mu}} \leq \min _{i \in \mathcal{N}}\left\{\frac{\beta_{1}(i)}{\sigma_{1}^{2}(i)}\right\}$, it holds

$$
\begin{aligned}
\mathcal{L} V_{1} \leq & \beta_{1}\left(\Lambda_{t}\right)\left(\frac{N(0) e^{-\hat{\mu} t}}{\alpha_{1}}+\frac{\check{A}}{\alpha_{1} \hat{\mu}}\right)-\left[\mu\left(\Lambda_{t}\right)+d_{1}\left(\Lambda_{t}\right)+r_{1}\left(\Lambda_{t}\right)\right] \\
& -\frac{\sigma_{1}^{2}\left(\Lambda_{t}\right)}{2}\left(\frac{N(0) e^{-\hat{\mu} t}}{\alpha_{1}}+\frac{\check{A}}{\alpha_{1} \hat{\mu}}\right)^{2} \\
\leq & \frac{\beta_{1}\left(\Lambda_{t}\right) \check{A}}{\alpha_{1} \hat{\mu}}-\left[\mu\left(\Lambda_{t}\right)+d_{1}\left(\Lambda_{t}\right)+r_{1}\left(\Lambda_{t}\right)\right]-\frac{\sigma_{1}^{2}\left(\Lambda_{t}\right)}{2}\left(\frac{\check{A}}{\alpha_{1} \hat{\mu}}\right)^{2}+c e^{-\hat{\mu} t}
\end{aligned}
$$

for a certain constant $c$. Substituting it into (22), integrating for the both sides from 0 to $t$ and dividing by $t$, we can obtain that

$$
\begin{aligned}
\frac{\ln I_{1}(t)}{t} \leq & \frac{1}{t} \int_{0}^{t}\left\{\frac{\beta_{1}\left(\Lambda_{s}\right) \check{A}}{\alpha_{1} \hat{\mu}}-\left[\mu\left(\Lambda_{s}\right)+d_{1}\left(\Lambda_{s}\right)+r_{1}\left(\Lambda_{s}\right)\right]-\frac{\sigma_{1}^{2}\left(\Lambda_{s}\right)}{2}\left(\frac{\check{A}}{\alpha_{1} \hat{\mu}}\right)^{2}\right\} d s \\
& +\frac{1}{t} \int_{0}^{t} c e^{-\hat{\mu} s} d s+\frac{M(t)}{t}+\frac{\ln I_{1}(0)}{t} .
\end{aligned}
$$

This, along with Lemmas 3.2 and 3.3, yields

$$
\limsup _{t \rightarrow \infty} \frac{\ln I_{1}(t)}{t} \leq \sum_{i \in \mathcal{N}} \pi(i)\left[\frac{\beta_{1}(i) \check{A}}{\alpha_{1} \hat{\mu}}-\left[\mu(i)+d_{1}(i)+r_{1}(i)\right]-\frac{\sigma_{1}^{2}(i)}{2}\left(\frac{\check{A}}{\alpha_{1} \hat{\mu}}\right)^{2}\right],
$$

where we use the result that

$$
\limsup _{t \rightarrow \infty} \frac{1}{t} \int_{0}^{t} c e^{-\hat{\mu} s} d s=\limsup _{t \rightarrow \infty} \frac{c}{\hat{\mu} t}\left(1-e^{-\hat{\mu} t}\right)=0 .
$$

Under the assumption of condition (17), we can get that the disease $I_{1}(t)$ will go to extinction.

Applying Itô's formula to $V_{2}=\ln I_{2}(t)$, one has

$$
d \ln I_{2}(t)=\mathcal{L} V_{2} d t+\frac{\sigma_{2}\left(\Lambda_{t}\right) S(t)}{\alpha_{2}+S(t)} d B_{2}(t)
$$

where $\mathcal{L} V_{2}=\frac{\beta_{2}\left(\Lambda_{t}\right) S(t)}{\alpha_{2}+S(t)}-\left[\mu\left(\Lambda_{t}\right)+d_{2}\left(\Lambda_{t}\right)+r_{2}\left(\Lambda_{t}\right)\right]-\frac{\sigma_{2}^{2}\left(\Lambda_{t}\right) S^{2}(t)}{2\left(\alpha_{2}+S(t)\right)^{2}}$.

According to the similar calculation above, and using the inequality $\frac{a}{a+b} \leq \frac{a+x}{a+b+x}$ for $a, b, x>0$, we get that, for some constant $c_{2}$,

$$
\begin{aligned}
\mathcal{L} V_{2} \leq & \beta_{2}\left(\Lambda_{t}\right)\left(\frac{N(0) e^{-\hat{\mu} t}+\frac{\check{A}}{\hat{\mu}}}{\alpha_{2}+N(0) e^{-\hat{\mu} t}+\frac{\check{A}}{\grave{\mu}}}\right)-\left[\mu\left(\Lambda_{t}\right)+d_{2}\left(\Lambda_{t}\right)+r_{2}\left(\Lambda_{t}\right)\right] \\
& -\frac{\sigma_{2}^{2}\left(\Lambda_{t}\right)}{2}\left(\frac{N(0) e^{-\hat{\mu} t}+\frac{\check{A}}{\hat{\mu}}}{\alpha_{2}+N(0) e^{-\hat{\mu} t}+\frac{\check{A}}{\grave{\mu}}}\right)^{2} \\
\leq & \frac{\beta_{2}\left(\Lambda_{t}\right) \check{A}}{\alpha_{2} \hat{\mu}+\check{A}}-\left[\mu\left(\Lambda_{t}\right)+d_{2}\left(\Lambda_{t}\right)+r_{2}\left(\Lambda_{t}\right)\right]-\frac{\sigma_{2}^{2}\left(\Lambda_{t}\right)}{2}\left(\frac{\check{A}}{\alpha_{2} \hat{\mu}+\check{A}}\right)^{2}+c_{2} e^{-\hat{\mu} t}
\end{aligned}
$$


and

$$
\begin{aligned}
\frac{\ln I_{2}(t)}{t} \leq & \frac{1}{t} \int_{0}^{t}\left\{\frac{\beta_{2}\left(\Lambda_{s}\right) \check{A}}{\alpha_{2} \hat{\mu}+\check{A}}-\left[\mu\left(\Lambda_{s}\right)+d_{2}\left(\Lambda_{s}\right)+r_{2}\left(\Lambda_{s}\right)\right]-\frac{\sigma_{2}^{2}\left(\Lambda_{s}\right) \check{A}^{2}}{2\left(\alpha_{2} \hat{\mu}+\check{A}\right)^{2}}\right\} d s \\
& +\frac{1}{t} \int_{0}^{t} c e^{-\hat{\mu} s} d s+\frac{M_{1}(t)}{t}+\frac{\ln I_{2}(0)}{t} .
\end{aligned}
$$

Therefore, we can obtain that

$$
\limsup _{t \rightarrow \infty} \frac{\ln I_{2}(t)}{t} \leq \sum_{i \in \mathcal{N}} \pi(i)\left[\frac{\beta_{2}(i) \check{A}}{\alpha_{2} \hat{\mu}+\check{A}}-\left[\mu(i)+d_{2}(i)+r_{2}(i)\right]-\frac{\sigma_{2}^{2}(i) \check{A}^{2}}{2\left(\alpha_{2} \hat{\mu}+\check{A}\right)^{2}}\right]
$$

Owing to assumption (18), one has $\lim _{t \rightarrow \infty} I_{2}(t)=0$, a.s. These complete the proof of (19).

Thanks to (19), we have $\lim \sup _{t \rightarrow \infty}\left(I_{1}(t)+I_{2}(t)\right)=0$, a.s. Then, by Lemma 3.1, (20) holds. As a result of (9), one has

$$
\begin{aligned}
\frac{1}{t} \int_{0}^{t} \mu\left(\Lambda_{s}\right) S(s) d s= & \frac{1}{t} \int_{0}^{t}\left[A\left(\Lambda_{s}\right)-\left(\mu\left(\Lambda_{s}\right)+d_{1}\left(\Lambda_{s}\right)\right) I_{1}(s)-\mu\left(\Lambda_{s}\right) R(s)\right] d s \\
& -\frac{1}{t} \int_{0}^{t}\left(\mu\left(\Lambda_{s}\right)+d_{2}\left(\Lambda_{s}\right)\right) I_{2}(s) d s-\frac{N(t)-N(0)}{t} .
\end{aligned}
$$

This, together with (19), (20), and Lemma 3.3, implies that conclusion (21) holds.

The proof is completed.

\section{Persistence of diseases}

In this section, we proceed to studying the conditions for persistence of diseases. Because there are two kinds of disease, we will discuss them respectively.

Theorem 4.1 Let $\left(S(t), I_{1}(t), I_{2}(t), R(t)\right)$ be the solution of model (2).

- Case 1. If $\frac{\check{A}}{\alpha_{2} \hat{\mu}+\check{A}} \leq \min _{i \in \mathcal{N}}\left\{\frac{\beta_{2}(i)}{\sigma_{2}^{2}(i)}\right\}, \Delta_{2}<1$, and

$$
\Delta_{3}:=\frac{\sum_{i \in \mathcal{N}} \pi(i) A(i)}{\kappa_{1} \sum_{i \in \mathcal{N}} \pi(i)\left[\mu(i)+d_{1}(i)+r_{1}(i)+\frac{\sigma_{1}^{2}(i) \check{A}^{2}}{2 \alpha_{1}^{2} \hat{\mu}^{2}}\right]}>1,
$$

where $\kappa_{1}=\alpha_{1} \max _{i \in \mathcal{N}}\left\{\frac{\mu(i)}{\beta_{1}(i)}\right\}$, then the infected individuals $I_{1}$ caused by disease 1 , or the disease $I_{1}$ for short, will be persistent in mean and the infected individuals $I_{2}$ caused by disease 2 , or the disease $I_{2}$ for short, will go to extinction.

- Case 2. If $\frac{\check{A}}{\alpha_{1} \hat{\mu}} \leq \min _{i \in \mathcal{N}}\left\{\frac{\beta_{1}(i)}{\sigma_{1}^{2}(i)}\right\}, \Delta_{1}<1$, and

$$
\Delta_{4}:=\frac{\sum_{i \in \mathcal{N}} \pi(i) A(i)}{\kappa_{2} \sum_{i \in \mathcal{N}} \pi(i)\left[\mu(i)+d_{2}(i)+r_{2}(i)+\frac{\sigma_{2}^{2}(i) \check{A}^{2}}{2\left(\alpha_{2} \hat{\mu}+\check{A}\right)^{2}}\right]}>1,
$$

where $\kappa_{2}=\frac{\hat{\mu} \alpha_{2}+\check{A}}{\hat{\mu}} \max _{i \in \mathcal{N}}\left\{\frac{\mu(i)}{\beta_{2}(i)}\right\}$, then the disease $I_{1}$ will go to extinction and disease $I_{2}$ will be persistent in mean. 
- Case 3. If $\Delta_{3}>1$ and $\Delta_{4}>1$, then the diseases $I_{1}, I_{2}$ will be persistent in mean. Moreover,

$$
\liminf _{t \rightarrow \infty}\left(\left\langle I_{1}(t)\right\rangle+\left\langle I_{2}(t)\right|\right) \geq \frac{1}{2 C}\left(N_{1}+N_{2}\right)
$$

where $N_{1}=\sum_{i \in \mathcal{N}} \pi(i)\left[A(i)-\kappa_{1}\left(\mu(i)+d_{1}(i)+r_{1}(i)+\frac{\sigma_{1}^{2}(i) \check{A}^{2}}{2 \alpha_{1}^{2} \hat{\mu}^{2}}\right)\right], N_{2}=\sum_{i \in \mathcal{N}} \pi(i)[A(i)-$ $\left.\kappa_{2}\left(\mu(i)+d_{2}(i)+r_{2}(i)+\frac{\sigma_{2}^{2}(i) \check{A}^{2}}{2\left(\alpha_{2} \hat{\mu}+\check{A}\right)^{2}}\right)\right]$.

Proof Case 1: We have proved the former part in Theorem 3.2, i.e., $\lim _{t \rightarrow \infty} I_{2}(t)=0$. Then, for $t$ sufficiently large and arbitrarily small $\epsilon, I_{2}(t)<\epsilon$. We just need to prove the second half, that is, $\frac{1}{t} \int_{0}^{t} I_{1}(s) d s>0$, when $\Delta_{3}>1$.

First, we prove that, for sufficiently large constant $C_{1}$,

$$
f(I, S, i):=\left[\left(\kappa_{1}-I\right) \frac{\beta_{1}(i)}{\alpha_{1}+I}-\mu(i)\right] S+C_{1} I \geq 0
$$

and

$$
g(I, S, i):=\left[\left(\kappa_{2}-I\right) \frac{\beta_{2}(i)}{\alpha_{2}+S}-\mu(i)\right] S+C_{2} I \geq 0,
$$

where $\kappa_{1}=\alpha_{1} \max _{i \in \mathcal{N}}\left\{\frac{\mu(i)}{\beta_{1}(i)}\right\}, \kappa_{2}=\frac{\hat{\mu} \alpha_{2}+\check{A}}{\hat{\mu}} \max _{i \in \mathcal{N}}\left\{\frac{\mu(i)}{\beta_{2}(i)}\right\}, I, S \in\left[0, N(0)+\frac{\check{A}}{\hat{\mu}}\right], i \in \mathcal{N}$.

Obviously, $f(0, S, i)=\left[\kappa_{1} \frac{\beta_{1}(i)}{\alpha_{1}}-\mu(i)\right] S$ and

$$
\begin{aligned}
\frac{\partial f}{\partial I} & =\left[-\frac{\beta_{1}(i)}{\alpha_{1}+I}-\left(\kappa_{1}-I\right) \frac{\beta_{1}(i)}{\left(\alpha_{1}+I\right)^{2}}\right] S+C_{1} \\
& \geq C_{1}-\left[\frac{\check{\beta_{1}}}{\alpha_{1}}+\kappa_{1} \frac{\check{\beta_{1}}}{\alpha_{1}^{2}}\right]\left(N(0)+\frac{\check{A}}{\grave{\mu}}\right) .
\end{aligned}
$$

For $\kappa_{1}=\alpha_{1} \max _{i \in \mathcal{N}}\left\{\frac{\mu(i)}{\beta_{1}(i)}\right\}$ and large constant $C_{1}, f(0, S, i) \geq 0$, and $\frac{\partial f}{\partial I}>0$. Hence, according to the continuity and monotonicity of $f, f(I, S, i) \geq 0$ for $I \in\left[0, I_{0}\right], S \in\left[0, N(0)+\frac{\check{A}}{\grave{\mu}}\right], i \in \mathcal{N}$.

We move forward to proving that $f(I, S, i) \geq 0$ when $I \in\left[I_{0}, N(0)+\frac{\check{A}}{\hat{\mu}}\right]$. From (27), we obtain that, for a sufficiently large constant $C_{1}$,

$$
f(I, S, i) \geq C_{1} I-\left[\frac{\beta_{1}(i) I}{\alpha_{1}+I}+\mu(i)\right] S \geq C_{1} I_{0}-\left(\check{\beta_{1}}+\check{A}\right)\left(N(0)+\frac{\check{A}}{\hat{\mu}}\right) \geq 0 .
$$

We have proved that (27) holds.

The proof of $g(I, S, i) \geq 0$ is similar to that of $f$. We omit it here.

Let $C=\max \left\{C_{1}, C_{2}\right\}$, then, for constant $C$, both $f \geq 0$ and $g \geq 0$ hold.

Next, from the first formula of (2) and utilizing the fact that $R(t) \geq 0$ and $I_{2}(t)<\epsilon$, we get that

$$
\begin{aligned}
d S(t) & \geq\left(A\left(\Lambda_{t}\right)-\mu\left(\Lambda_{t}\right) S(t)-\frac{\beta_{1}\left(\Lambda_{t}\right) S(t) I_{1}(t)}{\alpha_{1}+I_{1}(t)}-\check{\beta}_{2} \epsilon\right) d t-c(t) d B(t) \\
& =\left(A\left(\Lambda_{t}\right)-\frac{\kappa_{1} \beta_{1}\left(\Lambda_{t}\right) S(t)}{\alpha_{1}+I_{1}(t)}-C I_{1}(t)-\check{\beta}_{2} \epsilon+f\left(I_{1}, S, \Lambda_{t}\right)\right) d t-c(t) d B(t)
\end{aligned}
$$




$$
\geq\left(A\left(\Lambda_{t}\right)-\frac{\kappa_{1} \beta_{1}\left(\Lambda_{t}\right) S(t)}{\alpha_{1}+I_{1}(t)}-C I_{1}(t)-\check{\beta}_{2} \epsilon\right) d t-c(t) d B(t)
$$

where $c(t) d B(t)=c_{1}(t) d B_{1}(t)+c_{2}(t) d B_{2}(t), c_{1}(t)=\frac{\sigma_{1}\left(\Lambda_{t}\right) S(t) I_{1}(t)}{\alpha_{1}+I_{1}(t)}, c_{2}(t)=\frac{\sigma_{2}\left(\Lambda_{t}\right) S(t) I_{2}(t)}{\alpha_{2}+S(t)}$.

As a result of (22) and utilizing the result (28), one has

$$
\begin{aligned}
& d\left(\kappa_{1} \ln I_{1}(t)\right)=\kappa_{1} \mathcal{L} V_{1} d t+\kappa_{1} \frac{\sigma_{1}\left(\Lambda_{t}\right) S(t)}{\alpha_{1}+I_{1}(t)} d B_{1}(t) \\
& \geq\left(A\left(\Lambda_{t}\right)-C I_{1}(t)-\check{\beta}_{2} \epsilon-\kappa_{1}\left[\mu\left(\Lambda_{t}\right)+d_{1}\left(\Lambda_{t}\right)+r_{1}\left(\Lambda_{t}\right)\right]\right) d t-d S(t) \\
& \quad-\frac{\kappa_{1} \sigma_{1}^{2}\left(\Lambda_{t}\right) \check{A}^{2}}{2 \alpha_{1}^{2} \hat{\mu}^{2}} d t-c_{3} e^{-\hat{\mu} t} d t+\frac{\kappa_{1} \sigma_{1}\left(\Lambda_{t}\right) S(t)}{\alpha_{1}+I_{1}(t)} d B_{1}(t)-c(t) d B(t)
\end{aligned}
$$

for a certain constant $c_{3}$.

Integrating for both sides of (29) from 0 to $t$ and dividing by $t$, we can obtain that

$$
\begin{aligned}
C\left|I_{1}(t)\right\rangle \geq & \frac{1}{t} \int_{0}^{t}\left\{A\left(\Lambda_{s}\right)-\kappa_{1}\left[\mu\left(\Lambda_{s}\right)+d_{1}\left(\Lambda_{s}\right)+r_{1}\left(\Lambda_{s}\right)+\frac{\sigma_{1}^{2}\left(\Lambda_{s}\right) \check{A}^{2}}{2 \alpha_{1}^{2} \hat{\mu}^{2}}\right]\right\} d s \\
& -\check{\beta}_{2} \epsilon-\frac{c_{3} \int_{0}^{t} e^{-\hat{\mu} s} d s}{t}-\frac{S(t)-S(0)}{t}-\kappa_{1} \frac{\ln I_{1}(t)-\ln I_{1}(0)}{t} \\
& +\frac{1}{t} \int_{0}^{t}\left(\kappa_{1}-I_{1}(s)\right) \frac{\sigma_{1}\left(\Lambda_{s}\right) S(s)}{\alpha_{1}+I_{1}(s)} d B_{1}(s)-\frac{1}{t} \int_{0}^{t} c_{2}(s) d B_{2}(s) .
\end{aligned}
$$

Taking the inferior limit of the above formula and taking Lemma 3.2, Lemma 3.3, and (7) into account, one has

$$
\liminf _{t \rightarrow \infty}\left\langle I_{1}(t)\right\rangle \geq \frac{1}{C} \sum_{i \in \mathcal{N}} \pi(i)\left[A(i)-\kappa_{1}\left(\mu(i)+d_{1}(i)+r_{1}(i)+\frac{\sigma_{1}^{2}(i) \check{A}^{2}}{2 \alpha_{1}^{2} \hat{\mu}^{2}}\right)\right]-\check{\beta}_{2} \epsilon .
$$

Letting $\epsilon \rightarrow 0$ and with the help of (24), thus, the conclusion that $I_{1}(t)$ is persistent is available.

Case 2: To proceed, we refer to the above methods and procedures to obtain that

$$
\begin{aligned}
d S(t) & \geq\left(A\left(\Lambda_{t}\right)-\mu\left(\Lambda_{t}\right) S(t)-\frac{\beta_{2}\left(\Lambda_{t}\right) S(t) I_{2}(t)}{\alpha_{2}+S(t)}-c_{4} \epsilon\right) d t-c(t) d B(t) \\
& =\left(A\left(\Lambda_{t}\right)-\frac{\kappa_{2} \beta_{2}\left(\Lambda_{t}\right) S(t)}{\alpha_{2}+S(t)}-C_{2} I_{2}(t)-c_{4} \epsilon+g\left(I_{2}, S, \Lambda_{t}\right)\right) d t-c(t) d B(t) \\
& \geq\left(A\left(\Lambda_{t}\right)-\frac{\kappa_{2} \beta_{2}\left(\Lambda_{t}\right) S(t)}{\alpha_{2}+S(t)}-C_{2} I_{2}(t)-c_{4} \epsilon\right) d t-c(t) d B(t),
\end{aligned}
$$

where $c_{4}=\frac{\check{\beta_{1}}\left(N(0)+\frac{\check{A}}{\grave{\mu}}\right)}{\alpha_{1}}$.

From the result of (23) and exploiting (30), we get that

$$
\begin{aligned}
d( & \left.\kappa_{2} \ln I_{2}(t)\right) \\
\quad= & \kappa_{2} \mathcal{L} V_{2} d t+\kappa_{2} \frac{\sigma_{2}\left(\Lambda_{t}\right) S(t)}{\alpha_{2}+S(t)} d B_{2}(t) \\
\quad \geq & {\left[A\left(\Lambda_{t}\right)-C_{2} I_{2}(t)-\kappa_{2}\left[\mu\left(\Lambda_{t}\right)+d_{2}\left(\Lambda_{t}\right)+r_{2}\left(\Lambda_{t}\right)\right]-\frac{\kappa_{2} \sigma_{2}^{2}\left(\Lambda_{t}\right) \check{A}^{2}}{2\left(\alpha_{2} \hat{\mu}+\check{A}\right)^{2}}\right] d t }
\end{aligned}
$$




$$
-c_{4} \epsilon d t-c_{5} e^{-\hat{\mu} t} d t-d S(t)-c_{1}(t) d B_{1}(t)+\left(\kappa_{2}-I_{2}(t)\right) \frac{\sigma_{2}\left(\Lambda_{t}\right) S(t)}{\alpha_{2}+S(t)} d B_{2}(t)
$$

for some constant $c_{5}$.

Then integrating from 0 to $t$ and dividing by $t$ for both sides yield

$$
\begin{aligned}
C_{2}\left\langle I_{2}(t)\right\rangle \geq & \frac{1}{t} \int_{0}^{t}\left\{A\left(\Lambda_{s}\right)-\kappa_{2}\left[\mu\left(\Lambda_{s}\right)+d_{2}\left(\Lambda_{s}\right)+r_{2}\left(\Lambda_{s}\right)+\frac{\sigma_{2}^{2}\left(\Lambda_{s}\right) \check{A}^{2}}{2\left(\alpha_{2} \hat{\mu}+\check{A}\right)^{2}}\right]\right\} d s \\
& -c_{4} \epsilon-\frac{c_{5} \int_{0}^{t} e^{-\hat{\mu} s} d s}{t}-\frac{S(t)-S(0)}{t}-\kappa_{2} \frac{\ln I_{2}(t)-\ln I_{2}(0)}{t} \\
& -\frac{1}{t} \int_{0}^{t} c_{1}(s) d B_{1}(s)+\frac{1}{t} \int_{0}^{t}\left(\kappa_{2}-I_{2}(s)\right) \frac{\sigma_{2}\left(\Lambda_{s}\right) S(s)}{\alpha_{2}+S(s)} d B_{2}(s) .
\end{aligned}
$$

Taking the inferior limit of the above formula, along with the result of (6), Lemma 3.2, Lemma 3.3, deduces that

$$
\liminf _{t \rightarrow \infty}\left\langle I_{2}(t)\right\rangle \geq \frac{1}{C_{2}} \sum_{i \in \mathcal{N}} \pi(i)\left[A(i)-\kappa_{2}\left(\mu(i)+d_{2}(i)+r_{2}(i)+\frac{\sigma_{2}^{2}(i) \check{A}^{2}}{2\left(\alpha_{2} \hat{\mu}+\check{A}\right)^{2}}\right)\right]-c_{4} \epsilon .
$$

Due to the arbitrariness of $\epsilon$ and the assumption of (25), we can obtain that $\liminf _{t \rightarrow \infty} \frac{1}{t} \int_{0}^{t} I_{2}(s) d s>0$, i.e., the disease $I_{2}$ is persistent in mean.

Case 3: At last, we discuss the situation of $\Delta_{3}>1$ and $\Delta_{4}>1$. Due to $\Delta_{3}>1$ and $\Delta_{4}>1$, we have $N_{1}>0, N_{2}>0$. From the first formula of (2), we have

$$
\begin{aligned}
d S \geq & \left(A\left(\Lambda_{t}\right)-2 \mu\left(\Lambda_{t}\right) S-\frac{\beta_{1}\left(\Lambda_{t}\right) S I_{1}}{\alpha_{1}+I_{1}}-\frac{\beta_{2}\left(\Lambda_{t}\right) S I_{2}}{\alpha_{2}+S}\right) d t-c(t) d B(t) \\
= & \left(A\left(\Lambda_{t}\right)-\frac{\kappa_{1} \beta_{1}\left(\Lambda_{t}\right) S}{\alpha_{1}+I_{1}}-\frac{\kappa_{2} \beta_{2}\left(\Lambda_{t}\right) S}{\alpha_{2}+S}-C I_{1}(t)-C I_{2}(t)\right) d t \\
& +\left[f\left(I_{1}, S, \Lambda_{t}\right)+g\left(I_{2}, S, \Lambda_{t}\right)\right] d t-c(t) d B(t) \\
\geq & \left(A\left(\Lambda_{t}\right)-\frac{\kappa_{1} \beta_{1}\left(\Lambda_{t}\right) S}{\alpha_{1}+I_{1}}-\frac{\kappa_{2} \beta_{2}\left(\Lambda_{t}\right) S}{\alpha_{2}+S}-C\left(I_{1}+I_{2}\right)\right) d t-c(t) d B(t) .
\end{aligned}
$$

Then applying Itô's formula to $V(t)=\kappa_{1} \ln I_{1}(t)+\kappa_{2} \ln I_{2}(t)$ yields

$$
\begin{aligned}
C\left(I_{1}+I_{2}\right) d t \geq & \left\{\frac{1}{2} A\left(\Lambda_{t}\right)-\kappa_{1}\left[\mu\left(\Lambda_{t}\right)+d_{1}\left(\Lambda_{t}\right)+r_{1}\left(\Lambda_{t}\right)+\frac{\sigma_{1}^{2}\left(\Lambda_{t}\right) \check{A}^{2}}{2 \alpha_{1}^{2} \hat{\mu}^{2}}\right]\right\} d t \\
& +\left\{\frac{1}{2} A\left(\Lambda_{t}\right)-\kappa_{2}\left[\mu\left(\Lambda_{t}\right)+d_{2}\left(\Lambda_{t}\right)+r_{2}\left(\Lambda_{t}\right)+\frac{\sigma_{2}^{2}\left(\Lambda_{t}\right) \check{A}^{2}}{2\left(\alpha_{2} \hat{\mu}+\check{A}\right)^{2}}\right]\right\} d t \\
& -d V(t)-d S(t)-c_{3} e^{-\hat{\mu} t} d t-c_{5} e^{-\hat{\mu} t} d t \\
& +\left(\kappa_{1}-I_{1}(t)\right) \frac{\sigma_{1}\left(\Lambda_{t}\right) S(t)}{\alpha_{1}+I_{1}(t)} d B_{1}(t)+\left(\kappa_{2}-I_{2}(t)\right) \frac{\sigma_{2}\left(\Lambda_{t}\right) S(t)}{\alpha_{2}+S(t)} d B_{2}(t) .
\end{aligned}
$$

Integrating on both sides, dividing by $t$, and taking the inferior limit, then (26) can be obtained.

Next, we will compare the values given in Theorem 3.2 and Theorem 4.1 to show that the conclusions are compatible, that is to say, there is no contradiction between the conclusions. 
Theorem 4.2 For the $\Delta_{1}, \Delta_{2}, \Delta_{3}, \Delta_{4}$ presented in Theorem 3.2 and Theorem 4.1, $\Delta_{1} \geq \Delta_{3}$ and $\Delta_{2} \geq \Delta_{4}$ hold. Especially, $\Delta_{1}=\Delta_{3}$ is established if and only if $\check{A}=\hat{A}, \check{\mu}=\hat{\mu}, \breve{\beta}_{1}=\hat{\beta}_{1}$, i.e., $A(i), \mu(i), \beta_{1}(i), i \in \mathcal{N}$ are fixed constants. Also, $\Delta_{2}=\Delta_{4}$ holds if and only if $\check{A}=\hat{A}$, $\check{\mu}=\hat{\mu}, \check{\beta}_{2}=\hat{\beta}_{2}$, i.e., $A(i), \mu(i), \beta_{2}(i), i \in \mathcal{N}$ are fixed constants.

Proof From (17) and (24), one has

$$
\begin{aligned}
\frac{\Delta_{1}}{\Delta_{3}} & =\frac{\sum_{i \in \mathcal{N}} \pi_{i} \beta_{1}(i) \frac{\check{A}}{\hat{\mu}} \max _{i \in \mathcal{N}}\left\{\frac{\mu(i)}{\beta_{1}(i)}\right\}}{\sum_{i \in \mathcal{N}} \pi_{i} A(i)} \\
& =\frac{\sum_{i \in \mathcal{N}} \pi_{i} \beta_{1}(i) \max _{i \in \mathcal{N}}\left\{\frac{\mu(i)}{\hat{\mu}} \frac{1}{\beta_{1}(i)}\right\}}{\sum_{i \in \mathcal{N}} \pi_{i}\left(\frac{A(i)}{\check{A}}\right)} \\
& \geq \frac{\sum_{i \in \mathcal{N}} \pi_{i}\left(\frac{\beta_{1}(i)}{\hat{\beta}_{1}}\right)}{\sum_{i \in \mathcal{N}} \pi_{i}\left(\frac{A(i)}{\check{A}}\right)} \geq 1 .
\end{aligned}
$$

It is obvious that $\Delta_{1}=\Delta_{3}$ holds when $\check{A}=\hat{A}, \check{\mu}=\hat{\mu}, \check{\beta_{1}}=\hat{\beta}_{1}$. Next, we demonstrate that the converse is true. From the result of (31), we can see if $\Delta_{1}=\Delta_{3}$, then $\beta_{1}(i)=\hat{\beta}_{1}$ and $A(i)=\check{A}, i \in \mathcal{N}$, that is, $\beta_{1}(i) \equiv \beta_{1}, A(i) \equiv A$. Consequently, it holds $\max _{i \in \mathcal{N}}\{\mu(i)\}=\hat{\mu}$, which means $\mu(i) \equiv \mu$.

The proof of $\Delta_{2} \geq \Delta_{4}$ resembles that of the above. We omit it to avoid duplication.

\section{Remark 4.1}

1. We say that Theorem 3.2 and Theorem 4.1 are compatible when $\Delta_{1} \geq \Delta_{3}$ holds, i.e., if $\Delta_{1}<1$, then $\Delta_{3}<1$ and the disease $I_{1}$ will be extinct by Theorem 3.2. If $\Delta_{3}>1$, then $\Delta_{1}>1$ and the disease $I_{1}$ will be extinct according to Theorem 4.1. The situation of $I_{2}$ when $\Delta_{2} \geq \Delta_{4}$ holds is similar to $I_{1}$.

2. Suppose that there is no switching, i.e., $N=1$, then

$$
\Delta_{1}=\Delta_{3}=\frac{\frac{\beta_{1} A}{\alpha_{1} \mu}}{\mu+d_{1}+r_{1}+\frac{\sigma_{1}^{2} A^{2}}{2 \alpha_{1}^{2} \mu^{2}}}, \quad \Delta_{2}=\Delta_{4}=\frac{\frac{A \beta_{1}}{\mu \alpha_{2}+A}}{\mu+d_{2}+r_{2}+\frac{\sigma_{1}^{2} A^{2}}{2\left(\alpha_{2} \mu+A\right)^{2}}} .
$$

Obviously, $\Delta_{1}>1$ is equivalent to $R_{1}^{*}>1$ and $\Delta_{2}>1$ is equivalent to $R_{2}^{*}>1$, where $R_{1}^{*}$, $R_{2}^{*}$ can be seen in [15]. Our results are consistent with theirs. Therefore, we generalize the model with multiple environments and obtain more general results.

\section{Influence of Markovian switching upon incidence rate functions}

This section proceeds to studying the influence of Markovian switching upon incidence functions in model (4) and gives the conditions to judge disease extinction.

Lemma 5.1 For any initial value $\left(S(0), I_{1}(0), I_{2}(0), R(0)\right) \in \mathbb{R}_{+}^{4}$, and $i_{0} \in \mathcal{N}$, there is a unique solution $\left(S(t), I_{1}(t), I_{2}(t), R(t)\right)$ for $t>0$ to model (4), and the solution lies in $R_{+}^{4}$ with probability 1. Furthermore, the solution satisfies the inequality

$$
N(t) \leq N(0) e^{-\hat{\mu} t}+\frac{\check{A}}{\hat{\mu}} \leq N(0)+\frac{\check{A}}{\hat{\mu}}, \quad \text { a.s. }
$$

The proof is similar to that of Lemma 2.1. We omit here. 
Theorem 5.1 For the solution $S(t), I_{1}(t), I_{2}(t), R(t)$ to model (4) with the initial value $S(0), I_{1}(0), I_{2}(0), R(0) \in R_{+}^{4}$, if

$$
\begin{aligned}
& \Delta_{5}:=\frac{\sum_{i \in \mathcal{N}} \pi(i) \beta_{1}(i)\left(\frac{\check{A}}{\bar{\mu}}\right)^{i}}{\alpha_{1} \sum_{i \in \mathcal{N}} \pi(i)\left[\mu(i)+d_{1}(i)+r_{1}(i)\right]}<1, \\
& \Delta_{6}:=\frac{\sum_{i \in \mathcal{N}} \pi(i) \beta_{2}(i)\left(\frac{\check{A}}{\bar{\mu}}\right)^{i}}{\alpha_{2} \sum_{i \in \mathcal{N}} \pi(i)\left[\mu(i)+d_{2}(i)+r_{2}(i)\right]}<1,
\end{aligned}
$$

then

$$
\lim _{t \rightarrow \infty} I_{i}(t)=0, \quad i=1,2 \text {, a.s. }
$$

Proof We prove the first equality. Let $V_{3}(t)=\ln I_{1}(t)$, then one has

$$
\begin{aligned}
\mathcal{L} V_{3}(t) & =\frac{\beta_{1}\left(\Lambda_{t}\right) S I_{1}^{\Lambda_{t}-1}}{\alpha_{1}+I_{1}}-\left[\mu\left(\Lambda_{t}\right)+d_{1}\left(\Lambda_{t}\right)+r_{1}\left(\Lambda_{t}\right)\right]-\frac{\sigma_{1}^{2}\left(\Lambda_{t}\right) S^{2} I_{1}^{\Lambda_{t}-2}}{2\left(\alpha_{1}+I_{1}\right)^{2}} \\
& \leq \frac{\beta_{1}\left(\Lambda_{t}\right) N(t)^{\Lambda_{t}}}{\alpha_{1}+I_{1}}-\left[\mu\left(\Lambda_{t}\right)+d_{1}\left(\Lambda_{t}\right)+r_{1}\left(\Lambda_{t}\right)\right] \\
& \leq \sum_{i=1}^{N} \frac{\beta_{1}(i) N(t)^{i}}{\alpha_{1}} \mathbf{1}_{\left\{\Lambda_{t}=i\right\}}-\left[\mu\left(\Lambda_{t}\right)+d_{1}\left(\Lambda_{t}\right)+r_{1}\left(\Lambda_{t}\right)\right] \\
& \leq \sum_{i=1}^{N} \frac{\beta_{1}(i)\left(N(0) e^{-\hat{\mu} t}+\frac{\check{A}}{\hat{\mu}}\right)^{i}}{\alpha_{1}} \mathbf{1}_{\left\{\Lambda_{t}=i\right\}}-\left[\mu\left(\Lambda_{t}\right)+d_{1}\left(\Lambda_{t}\right)+r_{1}\left(\Lambda_{t}\right)\right] \\
& =\sum_{i=1}^{N} \frac{\beta_{1}(i)\left(\frac{\check{A}}{\hat{\mu}}\right)^{i}}{\alpha_{1}} \mathbf{1}_{\left\{\Lambda_{t}=i\right\}}+c_{6} e^{-\hat{\mu} t}-\left[\mu\left(\Lambda_{t}\right)+d_{1}\left(\Lambda_{t}\right)+r_{1}\left(\Lambda_{t}\right)\right] \\
& =\frac{\beta_{1}\left(\Lambda_{t}\right)\left(\frac{\check{A}}{\hat{\mu}}\right)^{\Lambda_{t}}}{\alpha_{1}}+c_{6} e^{-\hat{\mu} t}-\left[\mu\left(\Lambda_{t}\right)+d_{1}\left(\Lambda_{t}\right)+r_{1}\left(\Lambda_{t}\right)\right]
\end{aligned}
$$

for a constant $c_{6}$. Then

$$
d \ln I_{1}(t) \leq \mathcal{L} V_{3}(t)+\frac{\sigma_{1}\left(\Lambda_{t}\right) S I^{\Lambda_{t}-1}}{\alpha_{1}+I_{1}} d B_{1}(t) .
$$

With the help of Lemma 3.2 and (33), we arrive at the conclusion $\lim _{t \rightarrow \infty} I_{1}(t)=0$. The proof of the second equality resembles that of the first one.

The proof is completed.

\section{Examples and simulations}

In this section, we present some examples and numerical simulations to reveal some different phenomena which are not available without switching.

Let $\left(\Lambda_{t}\right)_{t \geq 0}$ be a continuous time Markov chain with state space $\mathcal{N}=\{1,2\}$, and its $Q$ matrix is

$$
Q_{1}=\left(\begin{array}{cc}
-p & p \\
q & -q
\end{array}\right)
$$

Hence, its stationary distribution $\pi=\left(\pi_{1}, \pi_{2}\right)=\left(\frac{q}{p+q}, \frac{p}{p+q}\right)$. 


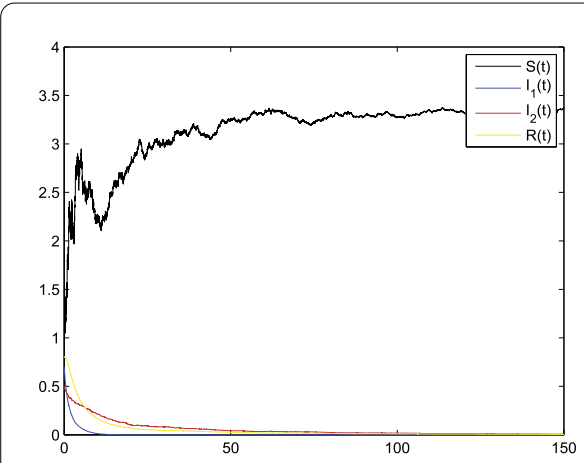

(a)

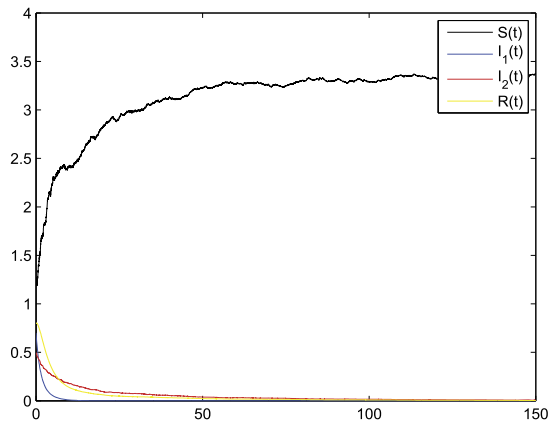

(b)

Figure 1 The trajectory of stochastic SIRS model (2) with parameters in Example 3 and the initial value $S(0)=1.2, I_{1}(0)=0.7, I_{2}(0)=0.5, R(0)=0.8$

Example 1 In order to prove the result of Theorem 3.1, we assume that $p=1, q=2, \alpha_{1}=1$, and $\alpha_{2}=1$. The values of other parameters are presented as follows:

$$
\begin{aligned}
& \left\{\begin{array} { l } 
{ A ( 1 ) = 0 . 3 } \\
{ A ( 2 ) = 0 . 4 , }
\end{array} \quad \left\{\begin{array} { l } 
{ \mu ( 1 ) = 0 . 1 } \\
{ \mu ( 2 ) = 0 . 0 8 , }
\end{array} \quad \left\{\begin{array} { l } 
{ d _ { 1 } ( 1 ) = 0 . 3 } \\
{ d _ { 1 } ( 2 ) = 0 . 2 , }
\end{array} \quad \left\{\begin{array}{l}
r_{1}(1)=0.4 \\
r_{1}(2)=0.5,
\end{array}\right.\right.\right.\right. \\
& \left\{\begin{array} { l } 
{ \beta _ { 1 } ( 1 ) = 0 . 1 5 } \\
{ \beta _ { 1 } ( 2 ) = 0 . 3 , }
\end{array} \quad \left\{\begin{array}{l}
\beta_{2}(1)=0.84 \\
\beta_{2}(2)=0.57
\end{array}\right.\right. \\
& \left\{\begin{array} { l } 
{ \sigma _ { 1 } ( 1 ) = 1 } \\
{ \sigma _ { 1 } ( 2 ) = 0 . 7 , }
\end{array} \quad \left\{\begin{array}{l}
\sigma_{2}(1)=0.8 \\
\sigma_{2}(2)=1
\end{array}\right.\right.
\end{aligned}
$$

Then (14) and (15) are satisfied, the diseases $I_{1}$ and $I_{2}$ will die out according to results of Theorem 3.1. Its simulation can be seen in Fig. 1(a).

Example 2 Next, we explain the results of Theorem 3.2. Assume that $p=1, q=3$, let $\sigma_{1}(1)=0.1, \sigma_{1}(2)=0.2, \sigma_{2}(1)=0.3$, and $\sigma_{2}(2)=0.2$, and other values of parameters are the same as those in (38). Then, by formulas 1 and (17), we obtain that ${ }_{(1)} R_{1}^{*}=0.5062<1$ in subsystem $1{ }_{(2)} R_{1}^{*}=1.2821>1$ in subsystem 2 , and $\Delta_{1}=0.9585<1$, which means that the disease $I_{1}$ in subsystem 1 goes to extinction and the disease $I_{1}$ in subsystem 2 is persistent, while owing to Markovian switching, the overall behavior of $I_{1}$ in model (2) will die out.

Let $d_{2}(1)=0.2, d_{2}(2)=0.35, r_{2}(1)=0.25, r_{2}(2)=0.4$, thus, by simple calculation, we get that ${ }_{(1)} R_{2}^{*}=1.0994>1$ in subsystem $1,_{(2)} R_{2}^{*}=0.5556<1$ in subsystem 2 , and $\Delta_{2}=0.9345<$ 1 , which means that the disease $I_{2}$ in subsystem 1 is persistent and the disease $I_{2}$ in subsystem 2 goes to extinction, while due to Markovian switching, the overall behavior of $I_{2}$ in model (2) will die out. Its trajectory can be seen in Fig. 1(b). 
Figure 2 The trajectory of stochastic SIRS model (2) with parameters in Example 3 and the initial value $S(0)$ $=1.2, I_{1}(0)=0.7, I_{2}(0)=0.5, R(0)=0.8$

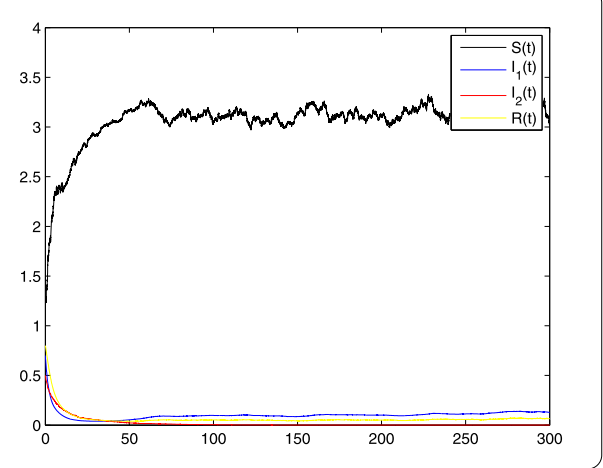

Example 3 To proceed, we give an example to illustrate the results of Case 1 in Theorem 4.1. Assume $p=8, q=1$. Let the values of parameters be given in the following form:

$$
\begin{aligned}
& \left\{\begin{array} { l } 
{ A ( 1 ) = 0 . 3 } \\
{ A ( 2 ) = 0 . 4 , }
\end{array} \quad \left\{\begin{array} { l } 
{ \mu ( 1 ) = 0 . 1 } \\
{ \mu ( 2 ) = 0 . 0 8 , }
\end{array} \quad \left\{\begin{array}{l}
d_{1}(1)=0.15 \\
d_{1}(2)=0.1,
\end{array}\right.\right.\right. \\
& \left\{\begin{array} { l } 
{ r _ { 1 } ( 1 ) = 0 . 3 } \\
{ r _ { 1 } ( 2 ) = 0 . 1 5 , }
\end{array} \quad \left\{\begin{array}{l}
d_{2}(1)=0.2 \\
d_{2}(2)=0.35,
\end{array}\right.\right. \\
& \left\{\begin{array} { l } 
{ r _ { 2 } ( 1 ) = 0 . 2 5 } \\
{ r _ { 2 } ( 2 ) = 0 . 4 , }
\end{array} \quad \left\{\begin{array}{l}
\beta_{1}(1)=0.2 \\
\beta_{1}(2)=0.1,
\end{array}\right.\right. \\
& \left\{\begin{array} { l } 
{ \beta _ { 2 } ( 1 ) = 0 . 8 4 } \\
{ \beta _ { 2 } ( 2 ) = 0 . 5 7 , }
\end{array} \quad \left\{\begin{array} { l } 
{ \sigma _ { 1 } ( 1 ) = 0 . 1 2 } \\
{ \sigma _ { 1 } ( 2 ) = 0 . 1 , }
\end{array} \quad \left\{\begin{array}{l}
\sigma_{2}(1)=0.3 \\
\sigma_{2}(2)=0.2 .
\end{array}\right.\right.\right.
\end{aligned}
$$

Then, by formula (24), we can obtain that ${ }_{(1)} R_{1}^{*}=0.9731<1,{ }_{(2)} R_{1}^{*}=1.1364>1, \Delta_{3}=$ $1.0011>1,{ }_{(1)} R_{2}^{*}=1.0994>1{ }_{(2)} R_{2}^{*}=0.5556<1$, and $\Delta_{2}=0.6137<1$. These values indicate that the disease of $I_{1}$ will become extinct in subsystem 1 and be persistent in subsystem 2, while the overall behavior of $I_{1}$, taking advantage of the results of Theorem 4.1, will be persistent. For the disease of $I_{2}$, it will continue in subsystem 1 and go to extinction in subsystem 2 , and the whole behavior, under the effect of Markovian switching, will die out. Its simulation can be seen in Fig. 2 .

Case 2 and Case 3 can be verified similarly.

Example 4 At last, we verify the results in Theorem 5.1. Assume $p=2, q=1$. Take the values of parameters as follows:

$$
\begin{array}{ll}
\left\{\begin{array}{l}
A(1)=0.3 \\
A(2)=0.25,
\end{array}\right. & \left\{\begin{array}{l}
\mu(1)=0.2 \\
\mu(2)=0.15,
\end{array}\right. \\
\left\{\begin{array} { l } 
{ r _ { 1 } ( 1 ) = 0 . 3 } \\
{ r _ { 1 } ( 2 ) = 0 . 4 , }
\end{array} \quad \left\{\begin{array}{l}
d_{2}(1)=0.1 \\
d_{2}(2)=0.05,
\end{array}\right.\right.
\end{array}
$$


Figure 3 The trajectory of stochastic SIRS model (4) with parameters in Example 4 and the initial value $S(0)$ $=1.2, I_{1}(0)=0.7, I_{2}(0)=0.5, R(0)=0.8$

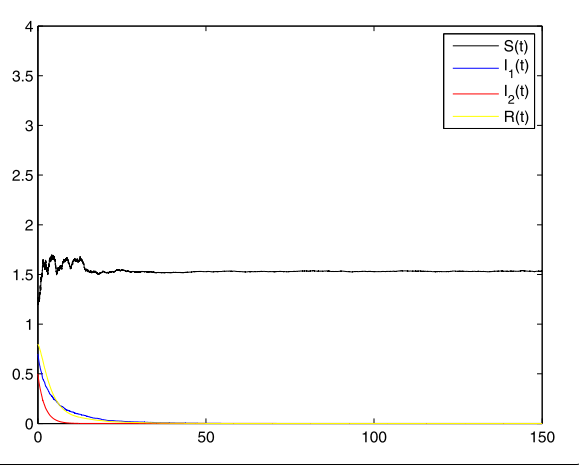

$$
\begin{aligned}
& \left\{\begin{array} { l } 
{ r _ { 2 } ( 1 ) = 0 . 4 } \\
{ r _ { 2 } ( 2 ) = 0 . 5 , }
\end{array} \quad \left\{\begin{array}{l}
\beta_{1}(1)=0.4 \\
\beta_{1}(2)=0.1,
\end{array}\right.\right. \\
& \left\{\begin{array} { l } 
{ \beta _ { 2 } ( 1 ) = 0 . 4 5 } \\
{ \beta _ { 2 } ( 2 ) = 0 . 1 3 , }
\end{array} \quad \left\{\begin{array} { l } 
{ \sigma _ { 1 } ( 1 ) = 0 . 1 2 } \\
{ \sigma _ { 1 } ( 2 ) = 0 . 1 , }
\end{array} \quad \left\{\begin{array}{l}
\sigma_{2}(1)=0.3 \\
\sigma_{2}(2)=0.2 .
\end{array}\right.\right.\right.
\end{aligned}
$$

Then (33) and (34) are satisfied. According to the result of Theorem 5.1, (35) holds. We can see its simulation in Fig. 3.

\section{Conclusions and future research}

In this paper, we have studied a stochastic SIRS model with two incidence rates and Markovian switching. We obtain that when the intensities of stochastic perturbation are large enough, the diseases will be extinct. Then the thresholds for judging the extinction or persistence of diseases are established by adopting appropriate inequality techniques when the intensities are small. We also prove that our conclusions are compatible. At last, we present some examples and simulations to verify our analytical results.

In addition, some other issues deserve to be further investigated. In this paper, the model with saturated incidence rates is studied, we can change the specific incidence functions into general ones in the future. Moreover, the models with state-dependent Markovian switching instead of state-independent regime switching in this paper can be further discussed. We leave these questions for our future work.

Funding

This work was supported by the National Natural Science Foundation of China (Nos: 11671404, 11661065) and the Fundamental Research Funds for the Central South University under grant No. $2018 z z t s 099$.

Competing interests

The authors declare that they have no competing interests.

Authors' contributions

Authors read and approved the final manuscript.

Author details

'School of Mathematics and Statistics, Central South University, Changsha, P.R. China. ${ }^{2}$ Department of Mathematics, Pingxiang University, Pingxiang, P.R. China.

Publisher's Note

Springer Nature remains neutral with regard to jurisdictional claims in published maps and institutional affiliations. 


\section{References}

1. Cai, Y., Yun, K., Wang, W.: A stochastic SIRS epidemic model with nonlinear incidence rate. Appl. Math. Comput. 305 221-240 (2017)

2. Guo, W., Zhang, Q., Li, X., Wang, W.: Dynamic behavior of a stochastic SIRS epidemic model with media coverage. Math. Methods Appl. Sci. 41(14), 5506-5525 (2018)

3. Lahrouz, A., Settati, A.: Necessary and sufficient condition for extinction and persistence of SIRS system with random perturbation. Appl. Math. Comput. 233(3), 10-19 (2014)

4. Liu, Q., Chen, Q., Jiang, D.: The threshold of a stochastic delayed SIR epidemic model with temporary immunity. Physica A 450, 115-125 (2016)

5. Liu, Z:: Dynamics of positive solutions to SIR and SEIR epidemic models with saturated incidence rates. Nonlinear Anal., Real World Appl. 14(3), 1286-1299 (2013)

6. Xu, C.: Global threshold dynamics of a stochastic differential equation SIS model. J. Math. Anal. Appl. 447(2), 736-757 (2016)

7. Zhao, Y., Jiang, D.: The threshold of a stochastic SIRS epidemic model with saturated incidence. Appl. Math. Lett. 34(1), 90-93 (2014)

8. Li, D., Liu, S., Cui, J.: Threshold dynamics and ergodicity of an SIRS epidemic model with Markovian switching. J. Differ. Equ. https://doi.org/10.1016/j.jde.2017.08.066(2017)

9. Guo, X., Luo, J.: Stationary distribution and extinction of SIR model with nonlinear incident rate under Markovian switching. Physica A 505, 471-481 (2018)

10. Bao, K., Zhang, Q.: Stationary distribution and extinction of a stochastic SIRS epidemic model with information intervention. Adv. Differ. Equ. 2017, 352 (2017)

11. Lahrouz, A., Omari, L.: Extinction and stationary distribution of a stochastic SIRS epidemic model with non-linear incidence. Stat. Probab. Lett. 83(4), 960-968 (2013)

12. Liu, Q., Jiang, D.: Stationary distribution and extinction of a stochastic SIR model with nonlinear perturbation. Appl. Math. Lett. 73, 8-15 (2017)

13. Meng, X.: Stability of a novel stochastic epidemic model with double epidemic hypothesis. Appl. Math. Comput. $217(2), 506-515(2010)$

14. Zhang, X., Jiang, D., Alsaedi, A., Hayat, T.: Stationary distribution of stochastic SIS epidemic model with vaccination under regime switching. Appl. Math. Lett. 59, 87-93 (2016)

15. Chang, Z., Meng, X., Lu, X.: Analysis of a novel stochastic SIRS epidemic model with two different saturated incidence rates. Physica A 472, 103-116 (2017)

16. Miao, A., Wang, X., Zhang, T., et al.: Dynamical analysis of a stochastic SIS epidemic model with nonlinear incidence rate and double epidemic hypothesis. Adv. Differ. Equ. 2017, 226 (2017)

17. Meng, X., Zhao, S., Tao, F., Zhang, T.: Dynamics of a novel nonlinear stochastic SIS epidemic model with double epidemic hypothesis. J. Math. Anal. Appl. 433(1), 227-242 (2015)

18. Bao, J., Shao, J.: Asymptotic behavior of SIRS models in state-dependent random environments (2018) arXiv:1802.02309

19. Economou, A., Lopez-Herrero, M.J.: The deterministic SIS epidemic model in a Markovian random environment. J. Math. Biol. 73(1), 91-121 (2016)

20. Gray, A., Greenhalgh, D., Mao, X., Pan, J.: The SIS epidemic model with Markovian switching. J. Math. Anal. Appl. 394(2), 496-516 (2012)

21. Greenhalgh, D., Liang, Y., Mao, X.: Modelling the effect of telegraph noise in the SIRS epidemic model using Markovian switching. Physica A 462, 684-704 (2016)

22. Settati, A., Lahrouz, A., Jarroudi, M.E., Jarroudi, M.E.: Dynamics of hybrid switching diffusions SIRS model. J. Appl. Math Comput. 52, 101-123 (2016)

23. Jin, M., Lin, Y., Pei, M.: Asymptotic behavior of a regime-switching SIR epidemic model with degenerate diffusion. Adv Differ. Equ. 2018, 84 (2018)

24. Hou, T., Shao, J.: Heavy tail and light tail of Cox-Ingersoll-Ross processes with regime-switching (2017) arXiv:1709.01691v1

25. Dang, N.H., Du, N.H., Yin, G.: Existence of stationary distributions for Kolmogorov systems of competitive type under telegraph noise. J. Differ. Equ. 257(6), 2078-2101 (2014)

26. Bao, J., Shao, J.: Permanence and extinction of regime-switching predator-prey models. SIAM J. Math. Anal. 48(1), 725-739 (2015)

27. Li, X., Gray, A., Jiang, D., Mao, X.: Sufficient and necessary conditions of stochastic permanence and extinction for stochastic logistic populations under regime switching. J. Math. Anal. Appl. 376(1), 11-28 (2011)

28. Li, X., Yin, G.: Logistic models with regime switching: permanence and ergodicity. J. Math. Anal. Appl. 441(2), 593-611 (2016)

29. Liu, M., Wang, K.: The threshold between permanence and extinction for a stochastic logistic model with regime switching. J. Appl. Math. Comput. 43(1-2), 329-349 (2013)

30. Mao, X., Yuan, C.: Stochastic Differential Equations with Markovian Switching. Imperial College Press, London (2006)

31. Yin, G.G., Zhu, C.: Hybrid Switching Diffusions: Properties and Applications. Springer, New York (2010) 